\title{
VESTIBOLOGY
}

\section{Towards a new staging of Ménière's disease: a vestibular approach}

\author{
Verso un nuovo staging della malattia di Ménière: un approccio vestibolare \\ O.A. SOBHY', D.M. ELMOAZEN¹, F.A. ABD-ELBAKY² \\ ${ }^{1}$ Audio-vestibular Unit, Department of Otorhinolaryngology, Faculty of Medicine, ${ }^{2}$ Department of \\ Otorhinolaryngology, Faculty of Medicine, Alexandria University, Alexandria, Egypt
}

\section{SUMMARY}

Ménière's disease (MD) is characterised by intermittent episodes of vertigo, fluctuating sensorineural hearing loss, tinnitus and aural pressure. Histopathologically, endolymphatic hydrops are seen initially in the cochlear duct and saccule, and with progression of the disease subsequent involvement of the utricle and semicircular canals occurs. The aims of this study are: 1) to assess the function of the saccule, utricle and three semicircular canals using cervical vestibular evoked myogenic potential (cVEMP), ocular VEMP (oVEMP) and video head impulse test (vHIT) in Ménière's patients, and 2) to correlate the findings with the different stages of MD. Forty patients diagnosed with unilateral definite MD according to the AAO-HNS (1995) criteria were tested. They were divided into four subgroups according to the stage of MD using the average of 500,1000, 2000 and $3000 \mathrm{~Hz}$ pure tone thresholds of the worst documented audiogram during the 6-month interval before examination. A control group of 40 healthy age-matched adults was equally divided into four corresponding subgroups according to their pure tone average. 500 and $1000 \mathrm{~Hz}$ tone burst air conduction cVEMP and oVEMP tests, bithermal caloric test and video HIT for all semicircular canals were recorded for both groups. The results of this study showed that the highest prevalence of abnormalities in Ménière's patients were seen in the PTA (85\%), followed by cVEMP (72.5\%) and oVEMP (67.5\%). Caloric test abnormalities followed with a lower prevalence of $32.5 \%$. The vHIT presented the lowest prevalence of abnormalities with $20 \%$ in the lateral canal, $7.5 \%$ in the anterior and $5 \%$ in the posterior. This pattern of abnormality agrees with the histopathological pattern of hydrops formation in the vestibular system. The findings of the current study suggest that with the advancement in the stage of MD, abnormal cVEMP, oVEMP, caloric and vHIT responses occur following the histopathological evidence of progression of the disease from the saccule up to the semicircular canals. Based on these findings, the authors suggest a new staging scheme for MD.

KEY WORDS: Cervical vestibular evoked myogenic potential $\bullet$ Ocular vestibular evoked myogenic potential • Video head impulse test • Ménière's disease • Staging of Ménière's disease

\section{RIASSUNTO}

La malattia di Ménière (MD) è caratterizzata da episodi intermittenti di vertigine, ipoacusia neurosensoriale fluttuante, tinnito e sensazione di ovattamento auricolare. L'idrope endolinfatica è inizialmente osservata a livello istopatologico nel dotto cocleare e nel sacculo, ma con la progressione della malattia anche l'utricolo e i canali semicircolari possono venire interessati. Lo scopo dello studio è duplice: 1) definire la funzione del sacculo, dell'utricolo e dei tre canali semicircolari per mezzo di potenziali evocati vestibolari miogeni cervicali e oculari (cVEMP, oVEMP) e del video-Head Impulse Test (vHIT) nei pazienti affetti da MD; 2) correlare $i$ risultati ottenuti con i diversi stadi di malattia. Quaranta pazienti con diagnosi di MD monolaterale secondo i criteri dell'AAO-HNS (1995) sono stati inclusi nello studio. I pazienti sono stati suddivisi in quattro sottogruppi, in base allo stadio di malattia e della soglia uditiva (definita dalla media delle frequenze 500, 1000, 2000, $3000 \mathrm{~Hz}$ ) ottenuta al peggiore esame audiometrico tonale osservato negli ultimi 6 mesi. Un gruppo di controllo di 40 soggetti adulti sani, equivalenti per età, è stato distribuito in modo uniforme in altrettanti quattro sottogruppi sulla base della soglia audiometrica. In entrambi i gruppi sono state ottenute le risposte di cVEMP, oVEMP, test calorico a stimolazione bitermica, vHIT per ciascuno dei canali semicircolari. I risultati mostrano nei pazienti affetti da MD una maggior prevalenza di alterazioni nel PTA (85\%), a seguire cVEMP (72,5\%) e oVEMP (67,5\%). Minor prevalenza di alterazioni sono state osservate per la stimolazione calorica $(32,5 \%)$ e il $v H I T$ con $20 \%$ per il canale semicircolare laterale, $7,5 \%$ per il superiore, $5 \%$ per il posteriore. Tale distribuzione di alterazioni funzionali rispecchia il pattern di danno istopatologico nel sistema vestibolare. In conclusione, con il progredire della MD si ottengono risposte patologiche ai test cVEMP, oVEMP, calorici e vHIT che riflettono la progressione istopatologica di malattia dal sacculo ai canali semicircolari. Sulla base di tali rilievi, gli autori propongono un aggiornato sistema classificativo per la MD.

PAROLE CHIAVE: cVEMP • oVEMP •Video Head Impulse Test • Malattia di Ménière • Stadiazione della malattia di Ménière 


\section{Introduction}

Ménière's disease (MD) is a clinical syndrome that consists of episodes of spontaneous vertigo, lasting several minutes to several days, usually associated with unilateral fluctuating sensorineural hearing loss, tinnitus and aural fullness. Histopathologically, the most notable feature is endolymphatic hydrops. Hydropic changes initially involve the cochlear duct and the saccule, linking the vestibular manifestations with hearing loss. However, with progression of the pathology, the entire endolymphatic space is involved with subsequent involvement of various endorgans including the utricle and semicircular canals (SCC) ${ }^{1}$. Schuknecht and Gulya reported a prevalence of endolymphatic hydrops of $100 \%$ in the cochlea, $86.3 \%$ in the saccule, $50 \%$ in the utricle and $36.4 \%$ in the SCCs based on pathological temporal bone studies ${ }^{2}$.

The assessment of patients with MD can be challenging, and the availability of investigations that examine each component of the vestibular apparatus has facilitated more complete evaluation. Tests for utricular and saccular function include ocular (oVEMP) and cervical (cVEMP) vestibular evoked myogenic potentials, respectively ${ }^{3}$. Caloric test with videonystagmography and the head impulse test have long been in clinical use as measures of the lateral SCC function. Lately, recordable video head impulse tests (vHIT) which measures vestibulo-ocular reflex (VOR) to rapid impulsive head accelerations in the plane of each SCC have allowed quantitative monitoring of individual canal function ${ }^{4}$. When the results of oVEMP and cVEMP are combined with the results of vHIT, a full understanding of the state of the peripheral vestibular function for each end organ of the labyrinth can be achieved with the progression of MD.

\section{Review of the current classifications/staging of MD}

- Shea (1993) staging ${ }^{5}$ (symptom-oriented).

- Stage I, cochlear stage: main symptoms: hearing loss and tinnitus;

- Stage II, some vestibular involvement: above symptoms + dizziness;

- Stage III, hydrops more extensive with obstruction of endolymph flow, permanent hearing loss, severe tinnitus and dizziness;

- Stage IV, hydrops fills labyrinth completely and endolymph pressure cannot rise, leading to subsidence of dizziness but unsteadiness remains, while hearing loss is more severe;

- Stage V, multiple ruptures in membranous labyrinth, no vertigo, only unsteadiness, hearing loss is profound or total, and the ear is lost.
- (AAO-HNS 1985 guidelines) ${ }^{6}$.

Four degrees of diagnostic staging were defined:

- possible MD: Episodic vertigo without documented HL, or SNHL (fluctuating or fixed) with disequilibrium;

- probable MD: one vertiginous episode lasting longer than $20 \mathrm{~min}$;

- definite MD: 2 or more definite spontaneous episodes of vertigo lasting longer than $20 \mathrm{~min}$, audiometrically documented hearing loss on at least one occasion and tinnitus or aural fullness in one ear;

- certain MD: Definite criteria plus histopathologic evidence of endolymphatic hydrops (at postmortem analysis).

- AAO-HNS, committee of hearing and equilibrium guidelines (1995) ${ }^{6}$.

This method of staging was purely audiometric. Based on pure tone average thresholds (PTA) at 500, 1000, 2000 and $3000 \mathrm{~Hz}$ of the worst audiogram in the last 6 months the following staging was suggested:

- stage I: PTA less than or equal to $26 \mathrm{~dB} \mathrm{HL}$;

- stage II: PTA 26-40 dB HL;

- stage III: PTA 41-70 dB HL;

- stage IV: PTA more than $70 \mathrm{~dB}$ HL.

The aforementioned staging protocols can be criticised based on the following:

- Shea staging: outdated since proposed therapies such as sac decompression and vestibular neurectomy are rarely resorted to now;

- AAO-HNS (1985): based on symptomatology and audiometry alone;

- AAO-HNS (1995): entirely audiometric (cochlear), but MD is a cochleo-vestibular disorder.

All above staging methods ignore:

1. the histopathologic spread of the disease from pars inferior to pars superior;

2. recent developments in vestibular diagnosis, namely the advent of VEMP and vHIT.

\section{Aim}

The objective of this study was to: 1) study the full audiovestibular profile in the different stages of MD in order to correlate this profile with the known histopathological progress of the disease, and 2) develop a new staging for the disease based on this profile.

\section{Subjects and methods}

Forty patients diagnosed with unilateral definite MD according to the American Academy of Otolaryngology and 
Head and Neck Surgery's 1995 criteria (AAO-HNS) were divided into four subgroups ${ }^{6}$. According to these criteria, patients should experience 2 or more spontaneous vertigo episodes lasting more than 20 min accompanied by tinnitus or fullness in one ear with audiometrically documented hearing loss on at least one occasion. The subgroups were divided according to the stage of MD using the AAO-HNS (1995) criteria based on 4 pure tone average at $0.5,1,2$ and $3 \mathrm{kHz}$ of the worst documented audiogram during the 6-month interval before examination. Stage I has a pure tone average less than $26 \mathrm{~dB}$ (6 patients), stage II between $26-40 \mathrm{~dB}$ (10 patients), stage III between 40-70 dB (15 patients) and stage IV more than $70 \mathrm{~dB}$ (9 patients). Patients were not examined in the acute attack and were instructed to cease their MD medication for 48 hours before the examination. The local ethics committee approved the study, and informed consent was obtained from each subject prior to inclusion. All patients had normal brain MRI. Patients with eye motility disorders, conductive hearing loss, evidence of MD in the other ear and any alternative cause of vertigo were excluded from the study. The control group consisted of 40 healthy age matched adults divided equally into four subgroups according to their pure tone average and were matching the audiograms of the four patient subgroups.

The following audio-vestibular test battery was carried out for each subject in the same day. Both affected and unaffected ears were tested. All patients were subjected to detailed history, frequency and duration of vertigo and disease duration.

\section{Pure tone audiometry}

Pure tone audiograms were obtained for each ear of all subjects using an Interacoustics AC 33 - audiometer. The 4 tone average thresholds at 500,1000, 2000 and $3000 \mathrm{~Hz}$ were calculated.

\section{Cervical VEMP}

Cervical VEMP (cVEMP) was recorded using the GSI AUDERA evoked potential system. Stimuli were airconducted 500 and $1000 \mathrm{~Hz}$ tone burst of condensation polarity and were monaurally delivered to the tested ear at fixed intensity of $105 \mathrm{~dB}$ nHL via GSI TIP50 insert earphones. Blackman gated 2-1-2 cycle stimuli were used at a rate of 5 cycles/sec. One channel recordings were obtained. The active electrode was placed on the upper third of the ipsilateral sternocleidomastoid muscle, the reference electrode on the sternoclavicular junction and the ground electrode on the forehead. Electrode impedance was typically $<5000 \mathrm{Ohms}$. The patient was tested in a sitting position with the head rotated away from the side of stimulation. Myogenic variability was minimised by the use of target markers on the examination room walls. At least 2 runs were performed and 150 sweeps were averaged for each run with analysis time of $50 \mathrm{msec}$. EMG signals were amplified and band-pass filtered between 5 and $750 \mathrm{~Hz}$ and were monitored to maintain background muscle activity.

The analysed parameters of the cVEMP were p13-n23 amplitude, p13 and n23 latency, frequency peak amplitude ratio $(1000 / 500 \mathrm{~Hz}$ FPA) of the same ear and interaural amplitude difference (IAD).

The IAD in Ménière's patients was calculated as follows;

$$
\mathrm{IAD}=[(\mathrm{Au}-\mathrm{Aa}) /(\mathrm{Au}+\mathrm{Aa})] * 100
$$

(Au and Aa represent the peak amplitude of the unaffected side and the affected side, respectively) ${ }^{7}$.

A positive value for the IAD in Ménière's patients indicated a reduced VEMP amplitude in the affected ear, while a negative value indicated augmented amplitude in the diseased ear. During statistical analysis, the sign of the IAD value in controls and MD patients was omitted and the mean and standard deviation (SD) of the absolute values were used.

\section{Ocular VEMP}

Similar stimulus parameters were used as in cVEMP. The contralateral eye to the tested ear was selected. Subjects were tested in a seated position and were asked to maintain an upward gaze at a fixed mark in the ceiling mounted at $30^{\circ}$ from the neutral gaze point according to Govender et al. ${ }^{8}$. The active electrode was placed just inferior to the centre of the lower lid margin and the reference electrode was $1 \mathrm{~cm}$ below and the ground electrode on the forehead. The n1-p1 amplitude of the oVEMP response, $\mathrm{n} 1$ and $\mathrm{p} 1$ latency and $1000 / 500 \mathrm{~Hz}$ FPA of the same ear and IAD were calculated using the aforementioned equations.

\section{Bithermal caloric test}

The bithermal caloric test was performed using GN Otometrics ICS Charter videonystagmography. The patient lied in the supine position with the head flexed $30^{\circ}$ upward ${ }^{9}$. Four irrigations were performed separately ( 2 for each ear) with water temperature of 30 and $44^{\circ} \mathrm{C}$ with at least $5 \mathrm{~min}$ of rest between irrigations. A unilateral weakness in the caloric response was quantified according to the conventional Jongkees' formula. A value $\geq 25 \%$ was considered pathological.

\section{Video HIT}

vHIT was done using a GNOtometrics ICS Impulse system in the lateral, left anterior right posterior (LARP) and right anterior left posterior (RALP) semi-circular canals 
(SCC) planes. A pair of lightweight, tightly fitting goggles was worn. A small nine axis motion sensor on the goggles measured the head movement in free space ${ }^{10}$. The subject was seated 1 metre from the targeted mark as recommended by Weber et al. ${ }^{10}$. For the horizontal plane, the target was directly in front of the patient and the head was tilted anteriorly by $30^{\circ}$ and unpredictably thrust to the left or right into a $15^{\circ}-20^{\circ}$ angle (Fig. 1). For the vertical planes, Migliaccio et al. modified procedure was used with gaze directed in the plane of head rotation to mainly elicit vertical eye movement for two-dimensional video pupil tracking ${ }^{11}$. The patient's head was rotated $30^{\circ}$ to the right (LARP test) or left (RALP test) while fixating on the target out of the corner of the eye for the gaze to be in the plane of the tested canals (Fig. 1). Briefly, abrupt forward and backward head impulses were given in the plane of the vertical canals that allow stimulation of the anterior and the posterior SCC, respectively. A total of 5 accepted head impulses in each direction for each plane were performed. The average gain of the VOR responses for each canal and the gain asymmetry (GA) were calculated as follows:

$$
\mathrm{GA}=[(\mathrm{Gc}-\mathrm{Gi}) /(\mathrm{Gc}+\mathrm{Gi})]^{*} 100 \%
$$

[Gc and Gi represent the gain for head thrust exciting the contralateral (unaffected) canal and the ipsilateral (affected) canal] ${ }^{12}$.

Abnormal overt and/or covert saccades were measured. The vHIT results were defined as abnormal, if canal gain or canal gain asymmetry were outside the normative range and/or when refixation saccades (overt and/or covert) were present.

\section{Statistical procedures}

The statistical evaluation of data was carried out using SPSS software. Mean \pm SD were recorded for each parameter. Chi square test was used for qualitative data. Paired t-test and Student t-test were used for comparing the control, affected and unaffected ears for normally distributed data. Comparison between two dependent means for non-parametric data was done by using the Wilcoxon signed ranks test, and for two independent means the Mann Whitney test was used. Comparisons across the four different subgroups of MD in VEMP, vHIT and caloric tests were done using F-test (ANOVA) for normally distributed data and the Kruskal Wallis test for non-parametric data. Correlation of disease duration with the stage of MD was done using Spearman's correlation. VEMP, vHIT and caloric test results were defined as abnormal if they fell outside the $95 \%$ limits (mean $\pm 2 \mathrm{SD}$ ) of normal hearing control data (subgroup I). Prevalence of abnormality in all tests were compared using McNemar's test. ROC curves were used to obtain cut-off values for diagnosis of MD using the 1000/500 HzFPA of the cVEMP and oVEMP. Statistical significance was set at $p \leq 0.05$.

\section{Results}

The patient group consisted of 80 ears (40 unaffected ears and 40 affected ears). The left ear was more commonly affected (52.5\%). 25 males and 15 females were tested with an age range between 21 and 69 years (mean $43.30 \pm 13.77)$. The control group consisted of 20 males and 20 females ( 80 ears) with a mean age of $40.82 \pm 10.68$ years (21-58 years).
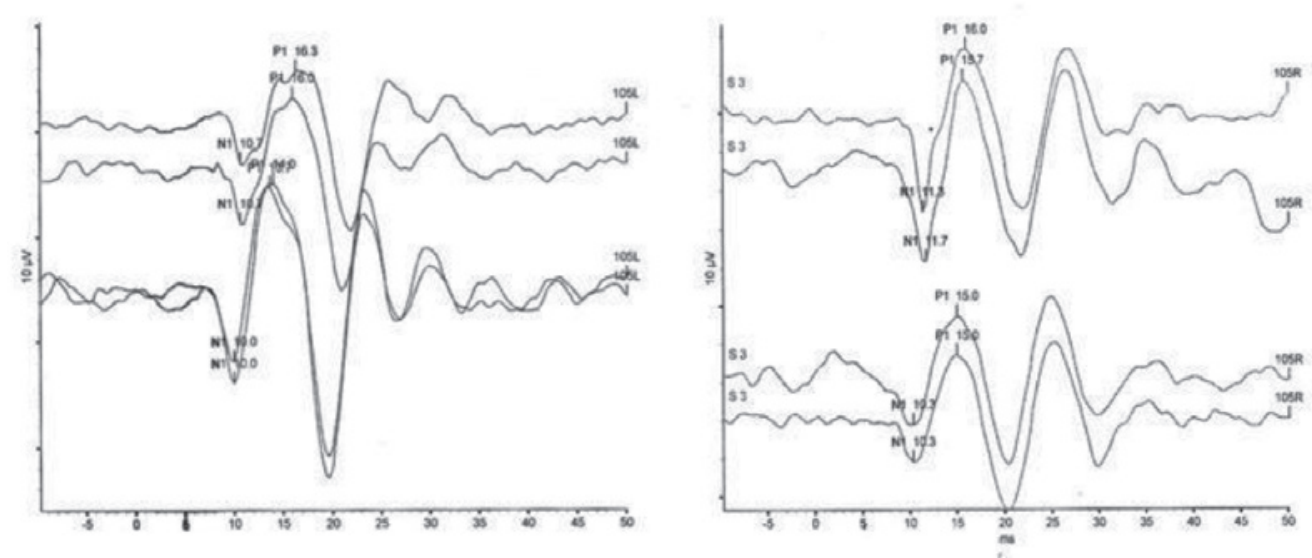

Fig. 1. Cervical VEMP waveforms in a Ménière's patient stage I with an affected left ear. The upper and lower waveforms are responses to $500 \mathrm{~Hz}$ and 1000 $\mathrm{Hz}$ stimuli, respectively. The right ear shows normal frequency tuning with the $500 \mathrm{~Hz}$ responses being slightly enhanced (right). The left ear shows altered frequency tuning with the $1000 \mathrm{~Hz}$ responses being greater in amplitude compared to the $500 \mathrm{~Hz}$ waveforms (left). 
Vertigo was the most common complaint (82\%) followed by tinnitus (77.5\%), fluctuating hearing loss $(77.5 \%)$ and ear fullness. In the earlier stages of MD, vertigo and vomiting were more pronounced, while dizziness was more evident in the late stages. Hearing loss was fluctuating in nature in the earlier stages of the disease with a low frequency configuration and accompanied by tinnitus and ear fullness. In the last stage of the disease, hearing loss became permanent with a flat configuration due to repeated rupture of membranes with progressive destruction of the membranous labyrinth ${ }^{13}$. The duration of illness ranged from 3 months to 15 years $(4.34 \pm 3.36)$ and the vertigo attacks lasted for $20 \mathrm{~min}$ to 2 hours $(33.88 \pm 19.98 \mathrm{~min})$. No significant correlation was found between disease duration and stage of disease.

Table 1 summarises the descriptive data (mean $\pm \mathrm{SD}$ ) of the measured indices of $500 \mathrm{~Hz}$ cVEMP, $500 \mathrm{~Hz}$ oVEMP and VHIT in subgroup I of controls, which represent the normative data and for the total population of controls, unaffected and affected ears of MD patients.

\section{Cervical VEMP results}

\section{a) Prevalence of cVEMP response}

The prevalence of cVEMP response to the $500 \mathrm{~Hz}$ and $1000 \mathrm{~Hz}$ air conduction stimuli were equal $(92.5 \%)$ in the control group, the affected ears of Ménière's patients had an insignificantly lower prevalence of $82.5 \%$ for both stimuli.

\section{b) Latency of cVEMP}

In response to the most commonly used $500 \mathrm{~Hz}$ stimulus, the mean latencies of cVEMP p13 and $\mathrm{n} 23$ waveforms were not significantly different in the affected ears compared to controls or the unaffected ears.

c) Amplitude of cVEMP

In the affected ears the $500 \mathrm{cVEMP}$ amplitude was normal in 10 ears $(25 \%)$, augmented in $8(20 \%)$, diminished in 15 $(37.5 \%)$ and absent in 7 (17.5\%). After exclusion of absent cVEMP ears, a significant difference was found between the mean amplitude of affected ears and controls, and also between unaffected and affected ears. No significance was found between control ears and unaffected ears of Ménière's patients. In subgroup I of MD patients, the mean cVEMP amplitude was significantly increased in affected ears $(508.58 \pm 234.40 \mu \mathrm{V})$ compared to unaffected ears (179.14 $\pm 154.66 \mu \mathrm{V})$ and control ears $(196.58 \pm 53.81 \mu \mathrm{V})$. In the other three subgroups (II-IV), the mean cVEMP amplitude was decreased significantly in affected ears compared to unaffected ears and control ears. Augmented VEMPs can be explained by dilatation of the saccular hydrops, pressing against the footplate and enhancing the sensitivity of the saccular macula to loud sounds ${ }^{14}$.
The mean $500 \mathrm{~Hz}$ cVEMP amplitude was compared across the four studied subgroups in controls and affected ears to determine the effect of hearing loss and progression of MD on the amplitude. No significant difference was found in the control group, thus excluding the effect of hearing loss on cVEMP amplitude. On the other hand, the affected ears of MD patients showed a significant difference between subgroup I and all other subgroups (IIIV). This can be attributed to the augmented amplitudes of subgroup I. A non-significant amplitude difference between subgroups II, III and IV of the affected ears of Ménière's patients was seen, although a trend towards a decrease in amplitude with the advancement of disease stage was observed.

d) Interaural Amplitude Difference of cVEMP

The absolute value of the IAD of the $500 \mathrm{~Hz}$ cVEMP had a significantly increased mean compared to controls using the entire sample and in each of the four subgroups, indicating increased interaural peak asymmetry in MD. With comparison across the subgroups no significant difference was found in the control group, while the affected ears of Ménière's patients showed a significant difference between most subgroups. In the current study, the suggested normal asymmetry limit (mean $\pm 2 \mathrm{SD}$ ) for the $500 \mathrm{~Hz}$ cVEMP IAD obtained from subgroup I of controls was $39.25 \%$.

e) Frequency tuning of cVEMP

In normal individuals, the cVEMP was shown to be most robustly elicited by using a $500 \mathrm{~Hz}$ tone-burst, with the response reducing on either side of this frequency. Thus, tuning curves may be potentially used as a method of diagnosing of MD. A significantly larger 1000/500 Hz FPA ratio was seen when comparing the entire sample of the affected ears of MD patients to control ears and unaffected ears. The increased FPA ratios denote the presence of altered tuning of ears affected by MD with shifting towards the higher $1000 \mathrm{~Hz}$ frequency (Fig. 1). Unaffected ears of MD showed no significant difference in 1000/500 Hz FPA compared to controls. A highly significant increased ratio in the affected ears was also seen in the four subgroups. Comparison across the subgroups in control ears and affected ears showed no significant differences demonstrating no effect of hearing loss on the tuning. ROC curves using the 1000/500 Hz FPA ratio of the cVEMP were used to obtain a cut-off value of $>0.9$ for diagnosis of MD, with $78.79 \%$ sensitivity and $80 \%$ specificity.

\section{Ocular VEMP results}

a) Prevalence of oVEMP response

The prevalence of oVEMP response to the $500 \mathrm{~Hz}$ and $1000 \mathrm{~Hz}$ air conduction stimuli in MD were similar 
$(92.5 \%)$ to the control group. The affected ears of Ménière's patients had a non-significantly lower prevalence of $80 \%$ using the $500 \mathrm{~Hz}$ stimulus compared to the $87.5 \%$ prevalence at $1000 \mathrm{~Hz}$ stimulus. The prevalence of both cVEMP and oVEMP responses together in affected ears showed a higher but non-significant prevalence for the $1000 \mathrm{~Hz}$ stimuli (90\%) compared to $500 \mathrm{~Hz}$ stimuli $(85 \%)$.

b) Latency of oVEMP

No significant difference was found in $500 \mathrm{~Hz}$ oVEMP $\mathrm{n} 1$ and $\mathrm{p} 2$ mean peak latency of affected, control or unaffected ears.

c) Amplitude of oVEMP

The 500 oVEMP amplitude of affected ears of Ménière's patients was normal in 23 ears $(57.5 \%)$, diminished in 9 $(22.5 \%)$ and absent in $8(20 \%)$. Unlike the cVEMP, no augmented oVEMP amplitude was obtained in any of the studied cases.

The oVEMP mean amplitude was significantly reduced in the affected ears of Ménière's patients compared to control ears and to the unaffected ears in the total sample and in comparing subgroups II, III and IV separately. No significant reduction in amplitude was found in the early stage of MD (subgroup I). Also, no significant difference was found between oVEMP amplitude in control ears and unaffected ears of Ménière's patients. Comparison across subgroups showed no significant difference in the control group, while the affected ears showed a significant difference between subgroup I and all other subgroups (II, III and IV).

d) Interaural Amplitude Difference of oVEMP

Ménière's patients showed significantly increased IAD compared to controls in subgroup III and IV only, indicating increased oVEMP asymmetry in the later stages of the disease. Comparison across subgroups showed no significant difference in the control group, while the affected ears showed significant difference in amplitude between earlier subgroups (I and II) and later subgroups (III and IV) and between subgroups III and IV. A pattern of increasing IAD with the increase in stage of the disease was seen .The calculated normal asymmetry limit for the 500 $\mathrm{Hz}$ IAD in oVEMP was $24.55 \pm 9.24 \%$.

e) Frequency tuning of the oVEMP

Similar to cVEMP results, a significantly larger 1000/500 Hz FPA ratio was seen in the affected ears of Ménière's patients compared to control and unaffected ears in the total sample and in subgroups II-IV. An example of the oVEMP frequency tuning is seen in Figure 2. Unaffected ears were non significantly different from controls. Comparison across the subgroups showed no significant difference in the control group or affected ears. The cutoff value for diagnosis of MD for oVEMP 1000/500 $\mathrm{Hz}$ FPA ratio was $>0.83$, with $90.32 \%$ sensitivity and $50 \%$ specificity. Other cut-off points were evaluated, but yielded unacceptably high false negative values.

\section{Bithermal caloric test results}

Caloric asymmetry was found in $32.5 \%$ of Ménière's patients with weakness ranging from 28.0-66.0\% (mean $43.23 \pm 12.58 \%$ ). Table II summarises the prevalence of abnormal caloric responses in stages I to IV.

\section{Video HIT results}

a) Semi-circular canal gain

The lateral canal gain was normal in 32 ears $(80 \%)$, increased in $3(7.5 \%)$ and diminished in $5(12.5 \%)$ (Table I). The anterior canal gain was normal in 38 ears (95\%) and diminished in $3(7.5 \%)$, while the posterior canal gain was
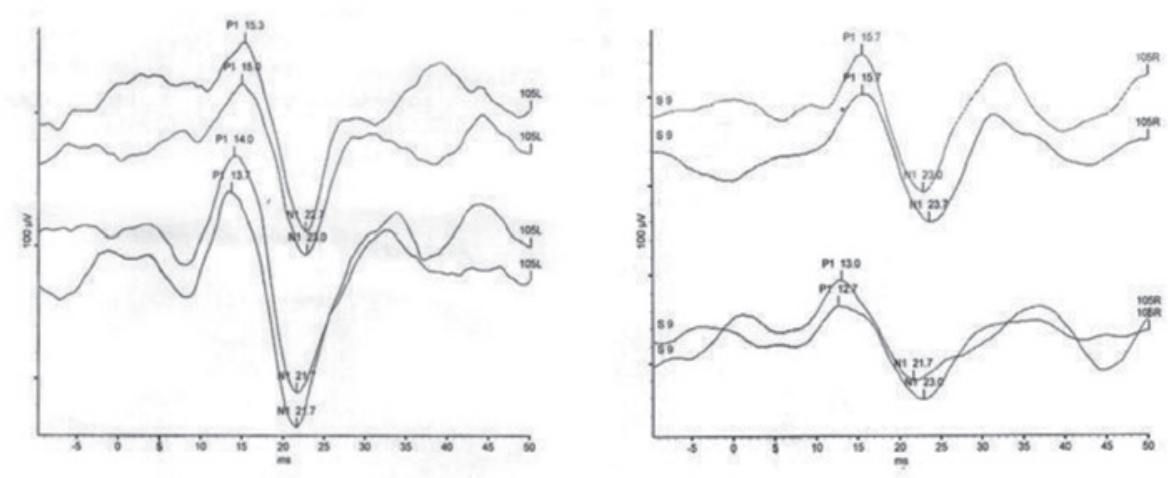

Fig. 2. Ocular VEMP waveforms in a Ménière's patient stage IV with affected left ear. The upper and lower waveforms are responses to $500 \mathrm{~Hz}$ and 1000 $\mathrm{Hz}$ stimuli, respectively. The right ear shows normal frequency tuning with the $500 \mathrm{~Hz}$ responses being slightly enhanced (right). The left ear shows altered frequency tuning with the $1000 \mathrm{~Hz}$ responses being greater in amplitude compared to the $500 \mathrm{~Hz}$ waveforms. 
Table I. Descriptive data (mean \pm SD) of vestibular tests indices in the total population and normative data.

\begin{tabular}{|c|c|c|c|c|c|}
\hline \multirow[t]{2}{*}{ Tests } & \multirow[t]{2}{*}{ Indices } & \multirow[t]{2}{*}{ Normative data } & \multicolumn{3}{|c|}{ Total population } \\
\hline & & & Control & Unaffected ears & Affected ears/Patients \\
\hline \multirow[t]{5}{*}{ cVEMP } & p13 latency (msec) & $15.62 \pm 0.85$ & $16.08 \pm 1.30$ & $16.16 \pm 1.05$ & $15.96 \pm 1.21$ \\
\hline & n23 latency (msec) & $24.99 \pm 1.97$ & $25.11 \pm 2.30$ & $24.98 \pm 1.95$ & $24.43 \pm 1.36$ \\
\hline & Amplitude $(\mu \mathrm{V})$ & $196.58 \pm 53.81$ & $204.58 \pm 71.31$ & $211.08 \pm 134.63$ & $201.48 \pm 197.82$ \\
\hline & $\operatorname{IAD}(\%)$ & $14.61 \pm 12.32$ & $13.84 \pm 7.50$ & - & $37.68 \pm 14.50$ \\
\hline & 1000/500 FPA & $0.86 \pm 0.27$ & $0.76 \pm 0.20$ & $0.76 \pm 0.36$ & $1.08 \pm 0.34$ \\
\hline \multirow[t]{5}{*}{ OVEMP } & n1 latency (msec) & $11.18 \pm 0.40$ & $11.47 \pm 0.79$ & $11.68 \pm 0.79$ & $11.88 \pm 1.17$ \\
\hline & p1 latency (msec) & $16.06 \pm 1.04$ & $16.66 \pm 1.71$ & $16.34 \pm 2.39$ & $17.10 \pm 1.66$ \\
\hline & Amplitude $(\mu \mathrm{V})$ & $10.98 \pm 4.50$ & $8.30 \pm 6.74$ & $7.22 \pm 4.46$ & $4.99 \pm 5.26$ \\
\hline & IAD (\%) & $24.55 \pm 9.24$ & $22.55 \pm 9.87$ & - & $29.92 \pm 15.69$ \\
\hline & $1000 / 500$ FPA & $1.02 \pm 0.53$ & $0.90 \pm 0.49$ & $0.81 \pm 0.35$ & $1.45 \pm 0.57$ \\
\hline \multirow[t]{3}{*}{ Canal gain } & Lateral canal & $1.03 \pm 0.12$ & $0.96 \pm 0.16$ & $0.99 \pm 0.14$ & $0.96 \pm 0.20$ \\
\hline & Anterior canal & $0.95 \pm 0.11$ & $0.95 \pm 0.20$ & $0.93 \pm 0.16$ & $1.01 \pm 0.21$ \\
\hline & Posterior canal & $1.03 \pm 0.18$ & $1.02 \pm 0.18$ & $1.0 \pm 0.18$ & $0.98 \pm 0.16$ \\
\hline \multirow{3}{*}{$\begin{array}{l}\text { Canal gain } \\
\text { asymmetry (\%) }\end{array}$} & Lateral canal & $8.72 \pm 4.63$ & $9.36 \pm 6.55$ & - & $8.0 \pm 6.46$ \\
\hline & Anterior canal & $11.54 \pm 7.85$ & $11.36 \pm 8.49$ & - & $11.01 \pm 8.64$ \\
\hline & Posterior canal & $10.75 \pm 10.03$ & $10.44 \pm 7.74$ & - & $8.07 \pm 7.32$ \\
\hline
\end{tabular}

Table II. Caloric response in the four studied subgroups of Ménière's patients.

\begin{tabular}{|c|c|c|c|c|c|c|c|c|}
\hline \multirow[b]{2}{*}{ Caloric response } & \multicolumn{2}{|c|}{ Subgroup I } & \multicolumn{2}{|c|}{ Subgroup II } & \multicolumn{2}{|c|}{ Subgroup III } & \multicolumn{2}{|c|}{ Subgroup IV } \\
\hline & No. & $\%$ & No. & $\%$ & No. & $\%$ & No. & $\%$ \\
\hline Normal & 6 & 100 & 8 & 80 & 8 & 53.3 & 5 & 55.6 \\
\hline Caloric weakness & 0 & 0 & 2 & 20 & 7 & 46.7 & 4 & 44.4 \\
\hline$<25$ & 6 & 100 & 8 & 80 & 8 & 53.3 & 5 & 55.6 \\
\hline $26-50$ & 0 & 0 & 2 & 20 & 6 & 40 & 2 & 22.2 \\
\hline$>50$ & 0 & 0 & 0 & 0 & 1 & 6.7 & 2 & 22.2 \\
\hline $\begin{array}{l}\text { Caloric weakness } \\
\text { Min.-Max. }\end{array}$ & \multicolumn{2}{|c|}{-} & \multicolumn{2}{|c|}{$33.0-40.0$} & \multicolumn{2}{|c|}{$28.0-54.0$} & \multicolumn{2}{|c|}{$35.0-66.0$} \\
\hline Mean \pm SD & \multicolumn{2}{|c|}{-} & \multicolumn{2}{|c|}{$36.50 \pm 4.95$} & \multicolumn{2}{|c|}{$41.0 \pm 9.80$} & \multicolumn{2}{|c|}{$50.50 \pm 17.90$} \\
\hline
\end{tabular}

normal in 37 ears $(92.5 \%)$ and diminished in $2(5 \%)$ with no increased responses in either canal. No refixation saccades, occult or overt, were recorded in any of the studied cases in any canal. No significant difference in gain was found between controls, unaffected and affected ears of Ménière's patients for all SCCs.

b) Canal gain asymmetry

The gain asymmetry of lateral canal was normal in 36 ears (90\%) and increased in 4 ears (10\%). No decreased gain asymmetry was seen in the lateral canal. The anterior and posterior canal gain asymmetries were normal in all 40 ears $(100 \%)$. No significant difference was found between control, affected and unaffected ears for the lateral, anterior and posterior SCCs. No effect of hearing loss on canal gain or asymmetry in control ears or affected ears was seen.

\section{Prevalence of abnormalities in MD}

Comparison of the prevalence of abnormalities in all the parameters of audio-vestibular tests performed in MD patients were done using McNemar's test. The highest prevalence in abnormalities in Ménière's patients were seen in the PTA $(85 \%)$ followed by cVEMP (72.5\%) and oVEMP $(67.5 \%)$. Caloric test abnormalities followed with a lower prevalence of $32.5 \%$ followed by vHIT lateral (20\%), anterior $(7.5 \%)$ and finally posterior (5\%) SCC abnormalities (Fig. 3). 


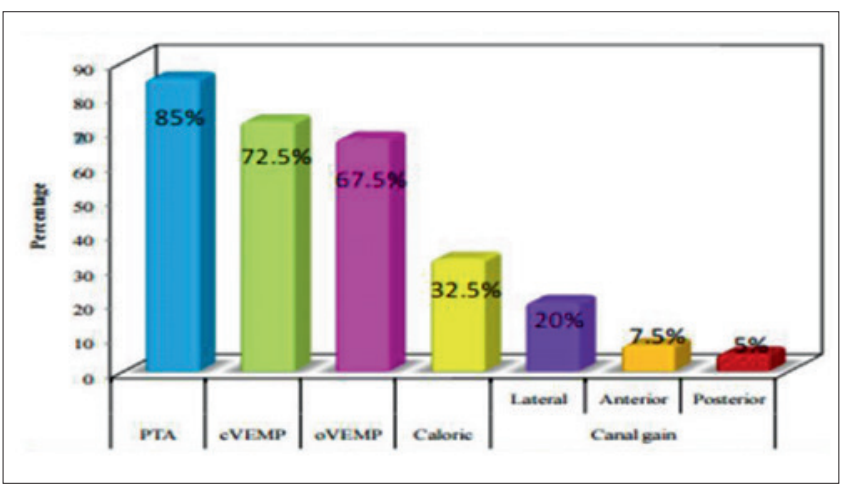

Fig. 3. The prevalence of abnormal test results in Ménière's disease.

\section{Discussion}

$\mathrm{MD}$ is associated with the accumulation of endolymph in the cochlear duct and vestibular organs. However, the presence of endolymphatic hydrops does not explain all clinical features, including progression of hearing loss and frequency of vertiginous attacks. Many researchers now believe that the abnormality related to Ménière's symptoms may be more physiological than obstructive anatomical. In such a context, MD would be the outcome of a complex cascade of pathophysiologic processes that ultimately dysregulate the ionic composition of the endolymph ${ }^{15}{ }^{16}$. As a consequence of impaired homeostasis, hearing and balance functions become vulnerable to internal and external factors, such as stress, sleep deprivation, etc.

Cervical and ocular VEMP abnormalities in the form of absent waveforms, reduced amplitude, elevated threshold, increased peak latencies, increased IAD, or altered frequency tuning in ears affected by MD have been reported in the literature 1718 .

In the current study, cVEMP amplitude showed a significantly enhanced response in stage I of the disease followed by a significant decrease in amplitude in the later stages. The IAD and 1000/500 Hz FPA ratio showed significantly increased values in all stages of the disease. Thus, the cVEMP test demonstrated abnormalities starting from stage I of MD indicating saccular affection early on in MD. The oVEMP parameters were not significantly different from those of controls in stage I of MD, denoting sparing of the utricle in the early stage of MD. In the later stages, significantly decreased amplitude and increased IAD and 100/500 Hz FPA ratio of oVEMP were seen.

Stage I of the disease did not show any caloric asymmetry. No significant difference was found between the stage of the disease and the prevalence or the degree of unilateral caloric weakness. However, a trend for an increase in the mean caloric asymmetry was seen with disease progression (Table II). The bithermal caloric test was reported to be asymmetrical in $28-73 \%$ of affected ears in MD in the previous literature ${ }^{719}$. Caloric unilateral weakness values are variable and weakness values of $>75 \%$ have been reported in some Ménière's patients with no correlation with the stage of disease ${ }^{7}$.

The recorded percentage of vHIT lateral canal gain asymmetry $(10 \%)$ were significantly lower than the percentage of unilateral caloric weakness $(32.5 \%)$ empathising the finding of the differential response of Ménière's ears to high and low frequency vestibular stimulation ${ }^{2021}$. In other words, selective loss of type II hair cells was thought to occur in Ménière's patients and was expected to result in selective loss of low frequency labyrinthine sensitivity, yielding poorer caloric test results than poorer vHIT results. The presumed preservation of type I hair cells was hence thought to preserve the high frequency sensitivity of the labyrinth necessary for the response to vHIT ${ }^{12}$. Nevertheless, Yacovino et al. were unable to detect higher than normal VOR gain in their single case of MD studied during the quiescent stage, which may suggest a fluctuating result of VOR gain in this disease ${ }^{22}$.

The three cases that showed increased gain may be explained by the relatively short 1 metre distance of the target that was adopted in this study following the recommendations of Weber et al. ${ }^{10}$. Other authors have recommended a distance more than 1 metre as VOR gain is a function of target distance, i.e. the shorter the distance the higher the gain ${ }^{23} 24$.

The vHIT gain and gain asymmetry of the three SCCs were insignificantly different from controls in all stages of the disease. Stage I of MD was free of vHIT abnormalities in all SCCs. Augmented gain responses were recorded only in the lateral canal in the last three stages. All three SCCs showed decreased gain and increased gain asymmetry in stages II-IV and were more frequently encountered in the lateral canal, while the posterior canal had the least percentage of abnormalities.

The recorded prevalence in abnormalities in Ménière's patients were in the PTA $>$ cVEMP $>$ oVEMP $>\mathrm{Ca}$ loric test $>$ lateral $\mathrm{SCC}>$ anterior $\mathrm{SCC}>$ posterior $\mathrm{SCC}$ (Fig. 3). This pattern of abnormality agrees with the reported pattern of hydrops formation in the vestibular system by Schuknecht and Gulya in pathological temporal bone studies ${ }^{2}$.

The percentage of abnormalities in the PTA (indicating cochlear abnormality) was not significantly higher than the cVEMP abnormalities pointing to saccular dysfunction accompanying the cochlear dyfunction. The PTA abnormalities were significantly higher than abnormali- 
ties in the oVEMP (utricular dyfunction), caloric test (lateral canal function at low frequencies) and vHIT (high frequency function of the three SCC). cVEMP abnormalities were significantly higher than the caloric test and the vHIT, while the caloric test was significantly higher than the anterior and posterior canals only. There was no significant difference in the prevalence of abnormalities between the three SCCs. These findings suggest consequent affection of the cochlea and saccule followed by the utricle then the low frequency function of the lateral SCC with the progression of MD. The involvement of higher frequency function of the lateral canal follows. The anterior and posterior canals are the least affected in MD.

The above findings prompted us to suggest a new staging system for MD, i.e. a system that considers that MD is a cochleo-vestibular disorder and not a mere cochlear disorder. Although there have been many proposals to classify $\mathrm{MD}$, the last of which by the Barany Society in $2015^{25}$, still there is a need for new staging of this disease.

The Barany Society classification suggested a twostaging system with definite and probable MD based on symptomatology, particularly the duration of vertigo and audiometry. The new proposed staging system in this paper utilises the vast audiological armamentarium of tools that specifically test each part of the audiovestibular apparatus. These tools, namely cVEMP, oVEMP and vHIT were not available 30 years ago when the old staging protocols were proposed. Finally, the new proposed staging system goes hand in hand with the known histopathology of MD.

Prevalence of endolymphatic hydrops in MD was reported as $100 \%$ in the cochlea, $86.3 \%$ in the saccule, $50 \%$ in the utricle and $36.4 \%$ in the semicircular canals ${ }^{26}$. The latter suggested that the disease progresses for pars inferior (cochlea) to pars superior (vestibule then SCC).

The authors suggest that the new staging could be as follows:

- stage (A): cochleo-saccular involvement with abnormal PTA and cVEMP;

- stage (B): cochleo-sacculo-utricular involvement with abnormal PTA, cVEMP and oVEMP;

- stage (C): cochlea-sacculo-utricular and lateral canal involvement with abnormal PTA, cVEMP, oVEMP and caloric test;

- stage (D): Entire labyrinthine involvement with abnormal PTA, cVEMP, oVEMP, caloric test and vHIT.

\section{Conflict of interest statement}

None declared.

\section{References}

1 Okuno T, Sando I. Localization, frequency, and severity of endolymphatic hydrops and the pathology of the labyrinthine membrane in Ménière's disease. Ann Otol Rhinol Laryngol 1987;96:438-45. https://doi.org/10.1177/000348948709600418

2 Schuknecht HF, Gulya AJ. Endolymphatic hydrops: an overview and classification. Ann Otol Rhinol Laryngol Suppl 1983;106:1-20.

3 Chiarovano E,Zamith F, Vidal PP, et al. Ocular and cervical VEMPs: a study of 74 patients suffering from peripheral vestibular disorders. Clin Neurophysiol 2011;122:1650-9. https://doi.org/10.1016/j. clinph.2011.01.006

4 Halmagyi GM, Aw ST, Cremer PD et al. Impulsive testing of individual semicircular canal function. Ann N Y Acad Sci 2001;942:192200. https://doi.org/10.1111/j.1749-6632.2001.tb03745.x

5 Shea JJ Jr. Classification of Ménière's disease. Am J Audiol 1993;14:224-9.

6 Committee on Hearing and Equilibrium guidelines for the diagnosis and evaluation of therapy in Menière's disease. American Academy of Otolaryngology-Head and Neck Foundation, Inc. Otolaryngol Head Neck Surg 1995;113:181-5. https://doi.org/10.1016/S01945998(95)70102-8

7 Young YH, Huang TW, Cheng PW. Assessing the stage of Ménière's disease using vestibular evoked myogenic potentials. Arch Otolaryngol Head Neck Surg 2003;129:815-8. https://doi.org/10.1001/ archotol.129.8.815

8 Govender S, Rosengren SM, Colebatch JG. The effect of gaze direction on the ocular vestibular evoked myogenic potential produced by air-conducted sound. Clin Neurophysiol 2009;120:1386-91. https:// doi.org/10.1016/j.clinph.2009.04.017

9 Bhansali SA, Honrubia V. Current status of electronystagmography testing. Otolaryngol Head Neck Surg 1999;120:419-26. https://doi. org/10.1016/S0194-5998(99)70286-X

10 Weber KP, MacDougall HG, Halmagyi GM, et al. Impulsive testing of semicircular canal function using video-oculography. Ann N Y Acad Sci 2009;1164:486-91. https://doi.org/10.1111/j.17496632.2008.03730.x

11 Migliaccio AA, Cremer PD. The 2D modified head impulse test: a $2 D$ technique for measuring function in all six semi-circular canals. J Vestib Res 2011;21:227-34. https://doi.org/10.3233/VES-20110421

12 Park HJ, Migliaccio AA, Della Santina CC, et al. Search-coil headthrust and caloric tests in Ménière's disease. Acta Otolaryngol 2005; 125:852-7.

13 Schuknecht HF, Ruther A. Blockage of longitudinal flow in endolymphatic hydrops. Eur Arch Otorhinolaryngol 1991;248:209-17.

14 van de Heyning PH, De Valck CFJ, Boudewyns A, et al. Ménière's disease. B-ENT 2007;3(Suppl 6):11-20.

15 Gates P. Hypothesis: could Ménière's disease be a channelopathy? Intern Med J 2005;35:488-9. https://doi.org/10.1111/j.14455994.2005.00891.x

16 Rauch SD. Clinical hints and precipitating factors in patients suffering from Ménière's disease. Otolaryngol Clin North Am 2010;43:1011-7. https://doi.org/10.1016/j.otc.2010.05.003

17 Sandhu JS, Low R, Rea PA, et al. Altered frequency dynamics of cervical and ocular vestibular evoked myogenic potentials in patients with Ménière's disease. Otol Neurotol 2012;33:444-9. https://doi. org/10.1097/MAO.0b013e3182488046

18 Winters SM, Campschroer T, Grolman W, et al. Ocular vestibular evoked myogenic potentials in response to air-conducted sound in Ménière's disease. Otol Neurotol 2011;32:1273-80. https://doi. org/10.1097/MAO.0b013e31822e5ac9 
19 Palomar-Asenjo V, Boleas-Aguirre MS, Sanchez-Ferrandiz N, et al. Caloric and rotatory chair test results in patients with Ménière's disease. Otol Neurotol 2006;27:945-50. https://doi.org/10.1097/01. mao.0000231593.03090.23

20 McGarvie LA, Curthoys IS, MacDougall HG, et al. What does the head impulse test versus caloric dissociation reveal about vestibular dysfunction in Ménière's disease? Ann N Y Acad Sci 2015;1343:5862. https://doi.org/10.1111/nyas.12687

21 Carey JP, Minor LB, Peng GC, et al. Changes in the three-dimensional angular vestibulo-ocular reflex following intratympanic gentamicin for Ménière's disease. J Assoc Res Otolaryngol 2002;3:43043. https://doi.org/10.1007/s101620010053

22 Yacovino DA, Hain TC, MusazziM. Fluctuating vestibulo-ocular reflex in Ménière's disease. Otol Neurotol 2017;38:244-47. https:// doi.org/10.1097/MAO.0000000000001298

23 ClémentG, Maciel F. Adjustment of the vestibulo-ocular reflex gain as a function of perceived target distance in humans. Neurosci Lett 2004;366:115-9. https://doi.org/10.1016/j.neulet.2004.05.022

${ }^{24}$ Castro P, Sena Esteves S, Lerchundi F, et al. Viewing target distance influences the vestibulo-ocular reflex gain when assessed using the video head impulse test. Audiol Neurotol 2018;23:285-89. https:// doi.org/10.1159/000493845

25 Lopez-Escamez JA, Carey J, Chung WH, et al.; Classification Committee of the Barany Society; Japan Society for Equilibrium Research; European Academy of Otology and Neurotology (EAONO); Equilibrium Committee of the American Academy of Otolaryngology-Head and Neck Surgery (AAO-HNS); Korean Balance Society. Diagnostic criteria for Menière's disease. J Vestib Res 2015;25:1-7. https://doi.org/10.3233/VES-150549

26 Oberman BS, Patel VA, Cureoglu S, et al. The aetiopathologies of Ménière's disease: a contemporary review. Acta Otorhinolaryngol Ital 2017;37:250-63. https://doi.org/10.14639/0392-100X-793

Received: December 13, 2018 - Accepted: June 13, 2019

How to cite this article: Sobhy OA, Elmoazen DM, Abd-Elbaky FA, et al. Towards a new staging of Ménière's disease: a vestibular approach. Acta Otorhinolaryngol Ital 2019;39:419-428. https://doi.org/10.14639/0392-100X-2461

Address for correspondence: Ossama A. Sobhy, 9 Ibrahim El-Sherif, Mostafa Kamel, Alexandria, Egypt. Tel. 0020122240247. E-mail: o.sobhy@ hotmail.com 